\title{
Low-density lipoprotein cholesterol
} outcomes post-non-PCSK9i lipid-lowering therapies in atherosclerotic cardiovascular disease and probable heterozygous familial hypercholesterolemia patients

This article was published in the following Dove Press journal: Therapeutics and Clinical Risk Management

\author{
Chi-Chang Chen' \\ Pallavi B Rane \\ Dionne M Hines' \\ Jeetvan Patel $^{2}$ \\ David J Harrison ${ }^{2}$ \\ Rolin L Wade \\ 'IQVIA, Plymouth Meeting, PA, USA; \\ ${ }^{2}$ Amgen Inc., Thousand Oaks, CA, USA
}

Correspondence: Pallavi B Rane Amgen Inc., I Amgen Center Dr, Thousand Oaks, CA 91320, CA, USA

Tel + I 8054475490

Email prane@amgen.com
Background: This study evaluated the proportion of patients with atherosclerotic cardiovascular disease (ASCVD) and probable heterozygous familial hypercholesterolemia (HeFH) achieving $\geq 50 \%$ reduction in low-density lipoprotein cholesterol (LDL-C) or reaching the LDL-C $\leq 70 \mathrm{mg} / \mathrm{dL}$ threshold, after initiating or modifying statin, and/or ezetimibe therapy.

Materials and methods: Adult ASCVD patients with baseline LDL-C $>70 \mathrm{mg} / \mathrm{dL}$ (index) and a subset of patients with probable $\mathrm{HeFH}$ (proxied by LDL-C $\geq 190 \mathrm{mg} / \mathrm{dL}$ ) were identified between January 1, 2012, and August 31, 2014, from the IQVIA electronic medical record database. Patients were followed for 12 months pre-index to examine baseline lipid-lowering therapy (LLT) use, and 12 months post index to evaluate treatment modifications and post-treatment LDL-C levels, stratified by type of treatment received and LDL-C levels at baseline.

Results: Of the sample of ASCVD patients who initiated treatment post-index $(\mathrm{n}=111,147)$, only $7.6 \%$ patients achieved a $\geq 50 \%$ reduction from baseline LDL-C and $19.1 \%$ of patients reached the LDL-C $\leq 70 \mathrm{mg} / \mathrm{dL}$ threshold. Among treated ASCVD patients who modified therapy post-index ( $\mathrm{n}=75,523), 5.6 \%$ achieved a $\geq 50 \%$ reduction in LDL-C, and proportion of patients achieving LDL-C $\leq 70 \mathrm{mg} / \mathrm{dL}$ ranged from $6.9 \%$ to $26.7 \%$, depending on the baseline LDL-C levels. Approximately $50 \%$ of the untreated probable $\mathrm{HeFH}$ patients $(\mathrm{n}=3,064)$ initiated LLT; however, the mean (SD) post-treatment LDL-C remained high (136.2 [47.8] mg/dL), with only $4.4 \%$ reaching LDL-C $\leq 70 \mathrm{mg} / \mathrm{dL}$. Of the treated probable HeFH patients $(\mathrm{n}=1,073)$, $41.5 \%$ modified treatment; $22.1 \%$ achieved a $\geq 50 \%$ reduction in LDL-C and $1.1 \%$ reached LDL-C $\leq 70 \mathrm{mg} / \mathrm{dL}$.

Conclusion: This study found that most patients had suboptimal LDL-C responses after initiating or modifying standard LLT (statin and/or ezetimibe). More frequent and aggressive lipid management, including increasing statin intensity and alternative therapies, may be needed in patients with ASCVD and probable HeFH to reduce their cardiovascular risk.

Keywords: hyperlipidemia, ASCVD, HeFH, LDL-C, statin, lipid-lowering therapy

\section{Introduction}

Cardiovascular disease (CVD) is the leading cause of death in the US, with more than 600,000 related deaths annually. ${ }^{1}$ Atherosclerotic cardiovascular disease (ASCVD) is a form of heart disease characterized by plaque build-up in the arteries that can lead to stroke, myocardial infarction (MI), and other life-threatening vascular events. ${ }^{2,3}$ 
Heterozygous familial hypercholesterolemia (HeFH) is an inherited lipid disorder associated with premature CVD. ${ }^{4,5}$ Elevated low-density lipoprotein cholesterol (LDL-C) is associated with increased cardiovascular (CV) risk and adverse clinical outcomes in both ASCVD and HeFH patients.

The American College of Cardiology and American Heart Association (ACC/AHA) 2013 guidelines suggested a 50\% reduction in LDL-C from baseline, while others, including prior version of the ACC/AHA guidelines, have suggested an LDL-C $\leq 70 \mathrm{mg} / \mathrm{dL}$ as a treatment target ${ }^{6}$ for ASCVD and HeFH patients. The 2013 ACC/AHA guidelines also recommended use of high-intensity statins for all patients with ASCVD or HeFH. Prior to the release of the 2013 guidelines, a number of studies reported that many patients failed to reach recommended LDL-C thresholds. ${ }^{7-11}$ Prior to the widespread availability of generic statins, 1 in 4 high-risk patients who were eligible for lipid-lowering therapy (LLT) remained untreated. ${ }^{7}$ It is not known if this has changed given the new guidelines, availability of generic statins, and expansion of insurance coverage with the Affordable Care Act.

The 2013 ACC/AHA cholesterol-lowering guidelines emphasize that treatment intensity should match the risk for adverse ASCVD events. ${ }^{6,10}$ This differs from previous guidelines that favored achieving LDL-C goal levels based on the estimated extent of CV risk. ${ }^{10,12}$ Statin agents are recommended as first-line therapy to manage ASCVD and HeFH; however, treatment with the cholesterol absorption inhibitor, ezetimibe, and/or a proprotein convertase subtilisin/kexin type 9 (PCSK9) inhibitor (such as evolocumab or alirocumab) may also be warranted in patients who cannot achieve adequate LDL-C control on a statin alone.,13-15 Evidence supporting statin therapy augmentation finds that adherence to statin therapy is suboptimal and that discontinuation rates are higher among patients prescribed high-intensity statin doses (eg, those at high CV risk and those with suboptimal response to a moderate-intensity dose). ${ }^{11,16-18}$ In addition, despite receiving statin therapy, patients at high CV risk are found to have an elevated 1-year risk of ASCVD-related re-hospitalization. ${ }^{11}$ To this point, the 2016 ACC/AHA expert consensus decision pathways describe the role of non-statin therapies in lowering LDL-C. ${ }^{19}$

The objective of this study was to evaluate the proportion of patients with ASCVD and subset of patients with ASCVD and probable HeFH (ASCVD + HeFH, proxied by a baseline LDL-C $>190 \mathrm{mg} / \mathrm{dL}$ ) with a baseline LDL-C $>70 \mathrm{mg} / \mathrm{dL}$ who reached an LDL-C threshold of $\leq 70 \mathrm{mg} / \mathrm{dL}$ and/or $\geq 50 \%$ reduction from baseline LDL-C after treatment initiation or modification.

\section{Materials and methods Data source}

The patient sample was drawn from the IQVIA US ambulatory electronic medical record (EMR) database containing approximately 47 million patient records from an "opt-in" provider research network. This aggregated database is comprised of records collected across 40,000 physicians in large practices and physician networks. Approximately $40 \%$ of contributing physicians are primary care practitioners and the remainder are specialists. IQVIA ambulatory EMR database has been used in previous retrospective observational studies. ${ }^{20-22}$ This study used data from January 1, 2011, to August 31, 2015; information on demographics, patient care episodes, risk factors, laboratory tests, diagnoses, procedures, and written prescriptions was included.

\section{Patient selection}

The study population was patients with at least a valid LDL-C lab value (ranging from 20 to $500 \mathrm{mg} / \mathrm{dL}$ ) between January 1 , 2012, and August 31, 2014. The first valid LDL-C value was considered a patient's baseline LDL-C and defined as their index date. Patients also had to have at least one recorded visit within the 12 months prior to their index date; evidence of ASCVD in the 12 months prior or on their index date; and at least 1 visit more than 12 months after their index date to ensure they were still active in the EMR. Patients with data quality issues, such as missing age, gender, or prescription data, were excluded. Eligible patients were followed for 12 months post-index.

The definition of ASCVD was based on the ACC/AHA definition and included MI, stroke, transient ischemic attack, peripheral artery disease, and stable or unstable angina. Patients with an ICD-9 diagnosis for any of these conditions prior to their first LDL-C assessment were categorized as having ASCVD. A subset of ASCVD patients were considered probable $\mathrm{HeFH}$ if the baseline LDL-C value was $\geq 190 \mathrm{mg} / \mathrm{dL}$ per the ACC/AHA 2016 expert consensus decision pathway. ${ }^{19}$

\section{Cohort definition}

Prior exposure to treatment was defined as a prescription order for a statin and/or ezetimibe. Patients were first categorized into one of four mutually exclusive baseline treatment cohorts based on their most recent prescriptions for statins or ezetimibe on or within 12 months before the index date: 1) untreated (no prescription order for a statin or ezetimibe); 2) statin only ( $\geq 1$ prescription for statin and no prescription for ezetimibe); 3 ) ezetimibe only 
( $\geq 1$ prescription for ezetimibe and no prescriptions for a statin); and 4) statin + ezetimibe ( $\geq 1$ prescription order for both a statin and ezetimibe, or $\geq 1$ prescription order for a statin/ezetimibe combination product). This study assumed that if orders for a statin and ezetimibe were both observed within 12 months pre-index or at index, they were used concurrently, and the patient was classified into the "statin + ezetimibe" cohort. Use of other lipid-lowering agents including niacin and bile acid sequestrates was not evaluated.

Patients' prior use of statins were further categorized and evaluated by statin dose intensity (low, moderate, high - see Table S1). The algorithm used to classify statin intensity was based on the 2013 ACC/AHA cholesterol guidelines. ${ }^{6}$

\section{Measures}

Patients were followed for 12 months post-index LDL-C assessment to evaluate their LDL-C outcomes, separately for those who initiated treatment and those who modified their prior LLT. Treatment initiation was use of any LLT in patients with no prior LLT treatment. Treatment modification was defined as switching statins, changing statin dose intensity, or augmenting current statin therapy with ezetimibe. "Switching" was defined as changing to a different statin while maintaining the same statin intensity level; "changing statin intensity" was defined as moving from one statin to another with a different intensity or changing the dose of their current statin where the new dose change resulted in a change in statin intensity; and "augmenting" was defined as adding ezetimibe to the statin regimen.

Demographic characteristics were measured on the index date including age, age group $(<18,18-44$, 45-54, 55-64, and $\geq 65$ years), gender, and geographic region (Northeast, South, Midwest, and West). Clinical characteristics included Charlson Comorbidity Index (CCI) score (Dartmouth-Manitoba adaptation), comorbidities (diabetes, hypertension, and dyslipidemia identified by using International Classification of Diseases, Ninth and Tenth Revision, Clinical Modification [ICD-9-CM and ICD-10 CM] diagnosis codes), baseline LDL-C, evidence of use of antidiabetic, antiarrhythmic, and antihypertensive therapy, body mass index, and specialty of treating physician (prescriber for baseline treatment or LDL-C lab test). Except for LDL-C, which was measured on the index date, clinical characteristics were assessed during the 12-month pre-index period, including the index date.

The primary outcome of interest was reaching LDL-C goal, defined as LDL-C of $\leq 70 \mathrm{mg} / \mathrm{dL}$ or a $\geq 50 \%$ reduction in LDL-C from baseline post-treatment initiation or modification. These measures were evaluated separately in patients who newly initiated statin and/or ezetimibe after index and in those who with prior statin or ezetimibe experienced a treatment modification during the post-index period. The first LDL-C value recorded 30 days to 12 months after treatment initiation or modification was used to determine attainment of LDL-C outcomes while allowing time for the new regimen to take effect. For all analyses, patients were further categorized into three groups by baseline LDL-C level: $71-100 \mathrm{mg} / \mathrm{dL}, 101-130 \mathrm{mg} / \mathrm{dL}$, and $>130 \mathrm{mg} / \mathrm{dL}$.

All analyses were conducted using SAS v9.2 (SAS Institute Inc., Cary, NC, USA) and no statistical comparisons were performed for this descriptive study. Outcomes were stratified by treatment cohort or index LDL-C (where applicable), and conducted separately for ASCVD and ASCVD + probable HeFH groups. The IQVIA ambulatory EMR database is a proprietary and HIPAA-compliant database. All patient data are de-identified, and no direct subject contact or primary collection of individual human subject data occurred. In addition, study results were in tabular form and aggregate analyses omitted subject identification; therefore, informed consent, ethics committee, or IRB approval were not required.

\section{Results Study subgroups}

A total of 4,431,936 patients with a valid LDL-C value during the study index period (January 1, 2012, to August $31,2014)$ and a diagnosis of ASCVD in the pre-index period were initially identified in the database. Of these, 260,607 $(5.9 \%)$ remained after applying other inclusion and exclusion criteria. For this study, the $71.6 \%(n=186,670)$ of patients with an uncontrolled baseline LDL-C $>70 \mathrm{mg} / \mathrm{dL}$ (Table 1) were selected for analysis. Of those, 50.5\% had an LDL-C 71-100 mg/dL, 29.2\% had an LDL-C 101-130 mg/dL; $20.3 \%$ had an LDL-C $>130 \mathrm{mg} / \mathrm{dL}$; and $2.2 \%$ had an LDL-C $\geq 190 \mathrm{mg} / \mathrm{dL}$ (probable HeFH patients; Table 2).

\section{Sample characteristics}

Among the 186,670 ASCVD patients with index LDL-C values $>70 \mathrm{mg} / \mathrm{dL}$, the proportion of males ranged from $40.9 \%$ in the LDL-C $>130 \mathrm{mg} / \mathrm{dL}$ group to $55.4 \%$ in the LDL-C 71-100 mg/dL group (Table 1). Mean (SD) ages among these subgroups decreased from 69.7 (10.8) years for the LDL-C 71-100 mg/dL group to 67.5 (11.9) years for the LDL-C 101-130 mg/dL group and 66.0 (12.0) years for the LDL-C $>130 \mathrm{mg} / \mathrm{dL}$ group. The largest group of patients resided in the US southern region (36.8\%). Aside from 
Table I All ASCVD sample: demographic and clinical characteristics

\begin{tabular}{|c|c|c|c|c|}
\hline \multirow{2}{*}{$\begin{array}{l}\text { Demographic and clinical } \\
\text { characteristics }\end{array}$} & \multicolumn{4}{|l|}{ All ASCVD, $N=186,670$} \\
\hline & $\begin{array}{l}\text { All ASCVD, index LDL-C } \\
\text { value }>70 \mathrm{mg} / \mathrm{dL} \\
\mathrm{N}=186,670(100 \%)\end{array}$ & $\begin{array}{l}\text { Index LDL-C value } \\
7 \mathrm{I}-100 \mathrm{mg} / \mathrm{dL} \\
\mathrm{n}=94,323(50.5 \%)\end{array}$ & $\begin{array}{l}\text { Index LDL-C value } \\
\text { I0 I-I } 30 \mathrm{mg} / \mathrm{dL} \\
\mathrm{n}=\mathbf{5 4 , 4 2 4}(\mathbf{2 9 . 2} \%)\end{array}$ & $\begin{array}{l}\text { Index LDL-C } \\
\text { value }>\text { I } 30 \mathrm{mg} / \mathrm{dL} \\
\mathrm{n}=\mathbf{3 7 , 9 2 3}(\mathbf{2 0 . 3 \% )}\end{array}$ \\
\hline Age in years, mean (SD) & $68.29(11.45)$ & $69.66(10.79)$ & $67.51(11.85)$ & $66.02(12.00)$ \\
\hline \multicolumn{5}{|l|}{ Age groups, \% } \\
\hline$<18$ & 0.0 & 0.1 & 0.1 & 0.0 \\
\hline $18-44$ & 0.8 & 2.4 & 4.1 & 4.8 \\
\hline $45-54$ & 2.6 & 7.3 & 10.7 & 13.3 \\
\hline $55-64$ & 9.5 & 18.7 & 21.4 & 23.9 \\
\hline$>65$ & 20.5 & 71.7 & 63.8 & 58.0 \\
\hline \multicolumn{5}{|l|}{ Gender, \% } \\
\hline Male & 50.3 & 55.4 & 48.0 & 40.9 \\
\hline Female & 49.7 & 44.6 & 52.1 & 59.1 \\
\hline \multicolumn{5}{|l|}{ Geographic region, \% } \\
\hline Northeast & 26.9 & 27.4 & 26.4 & 26.2 \\
\hline South & 36.8 & 35.8 & 37.4 & 38.5 \\
\hline Midwest & 20.6 & 20.7 & 20.5 & 20.7 \\
\hline West & 15.7 & 16.1 & 15.7 & 14.5 \\
\hline \multicolumn{5}{|l|}{$\mathrm{CCl}$ score, \% } \\
\hline 0 & 99.5 & 99.5 & 99.5 & 99.4 \\
\hline$>0$ & 0.5 & 0.5 & 0.5 & 0.6 \\
\hline Mean (SD) & $0.01(0.08)$ & $0.01(0.07)$ & $0.01(0.07)$ & $0.01(0.08)$ \\
\hline \multicolumn{5}{|l|}{ Comorbidities, \% } \\
\hline Diabetes & 27.3 & 30.1 & 25.0 & 23.8 \\
\hline Hypertension & 65.3 & 68.0 & 63.8 & 60.8 \\
\hline Dyslipidemia & 68.1 & 71.1 & 63.9 & 66.5 \\
\hline Coronary disease & 39.1 & 41.6 & 36.6 & 36.7 \\
\hline Cerebrovascular disease & 17.4 & 16.1 & 18.5 & 19.1 \\
\hline $\begin{array}{l}\text { Peripheral vascular } \\
\text { disease }\end{array}$ & 54.1 & 54.0 & 54.6 & 53.7 \\
\hline \multicolumn{5}{|l|}{ Index LDL-C, mg/dL } \\
\hline Mean (SD) & $108.12(31.60)$ & $84.73(8.52)$ & II3.8I (8.52) & I58.I2 (26.89) \\
\hline \multicolumn{5}{|l|}{ Number of LDL-C tests, \% } \\
\hline I (index test) & 43.5 & 43.6 & 44.6 & 41.6 \\
\hline 2 & 31.2 & 31.7 & 30.5 & 31.1 \\
\hline 3 & 14.6 & 14.5 & 14.4 & 15.3 \\
\hline 4 & 6.5 & 6.4 & 6.4 & 7.1 \\
\hline$>4$ & 4.1 & 3.8 & 4.2 & 5.0 \\
\hline \multicolumn{5}{|l|}{ Medications, \% } \\
\hline Antidiabetic & 17.2 & 19.0 & 15.8 & 14.9 \\
\hline Antiarrhythmic & 0.5 & 0.5 & 0.5 & 0.4 \\
\hline Antihypertensive & 59.9 & 62.6 & 58.6 & 55.4 \\
\hline \multicolumn{5}{|l|}{ Index BMI } \\
\hline Patients with BMI, \% & 91.0 & 90.9 & 91.1 & 91.0 \\
\hline Mean (SD) & $29.46(6.48)$ & $29.44(6.4 I)$ & $29.43(6.6 I)$ & $29.53(6.47)$ \\
\hline
\end{tabular}

Abbreviations: ASCVD, atherosclerotic cardiovascular disease; BMI, body mass index; CCl, Charlson Comorbidity Index; LDL-C, low-density lipoprotein cholesterol. 
Table 2 Probable HeFH sample: demographic and clinical characteristics

\begin{tabular}{|c|c|}
\hline \multirow{2}{*}{$\begin{array}{l}\text { Demographic and } \\
\text { clinical characteristics }\end{array}$} & Probable HeFH patients \\
\hline & $\begin{array}{l}\text { Index LDL-C value } \\
>190 \mathrm{mg} / \mathrm{dL} \\
\mathrm{n}=4,137(2.2 \%)\end{array}$ \\
\hline Age in years, mean (SD) & $65.32(11.57)$ \\
\hline \multicolumn{2}{|l|}{ Age groups, \% } \\
\hline$<18$ & 0.0 \\
\hline $18-44$ & 3.9 \\
\hline $45-54$ & 14.6 \\
\hline $55-64$ & 26.9 \\
\hline$>65$ & 54.7 \\
\hline \multicolumn{2}{|l|}{ Gender, \% } \\
\hline Male & 33.9 \\
\hline Female & 66.1 \\
\hline \multicolumn{2}{|l|}{ Geographic region, \% } \\
\hline Northeast & 25.7 \\
\hline South & 40.4 \\
\hline Midwest & 21.3 \\
\hline West & 12.6 \\
\hline \multicolumn{2}{|l|}{$\mathrm{CCl}$ score, \% } \\
\hline 0 & 99.2 \\
\hline$>0$ & 0.8 \\
\hline Mean (SD) & $0.01(0.09)$ \\
\hline \multicolumn{2}{|l|}{ Comorbidities, \% } \\
\hline Diabetes & 26.0 \\
\hline Hypertension & 62.7 \\
\hline Dyslipidemia & 72.0 \\
\hline Coronary disease & 40.2 \\
\hline Cerebrovascular disease & 18.2 \\
\hline Peripheral vascular disease & 52.5 \\
\hline \multicolumn{2}{|l|}{ Index LDL-C, mg/dL } \\
\hline Mean (SD) & $215.97(30.92)$ \\
\hline \multicolumn{2}{|l|}{ Number of LDL-C tests, \% } \\
\hline I (index test) & 38.0 \\
\hline 2 & 31.3 \\
\hline 3 & 16.7 \\
\hline 4 & 8.1 \\
\hline$>4$ & 5.9 \\
\hline \multicolumn{2}{|l|}{ Medications, \% } \\
\hline Antidiabetic & 16.3 \\
\hline Antiarrhythmic & 0.3 \\
\hline Antihypertensive & 56.2 \\
\hline \multicolumn{2}{|l|}{ Index BMI } \\
\hline Patients with BMI, \% & 90.8 \\
\hline Mean (SD) & $29.63(6.49)$ \\
\hline
\end{tabular}

Abbreviations: $\mathrm{BMI}$, body mass index; $\mathrm{CCl}$, Charlson Comorbidity Index; HeFH heterozygous familial hypercholesterolemia; LDL-C, low-density lipoprotein cholesterol. underlying ASCVD, the overall study population had few serious co-morbidities with over $99 \%$ patients in all LDL-C subgroups having a $\mathrm{CCI}=0$. However, a high proportion of patients in all LDL-C subgroups had comorbid hypertension (65.3\%), and most patients used antihypertensives, ranging from $62.6 \%$ of those in the $71-100 \mathrm{mg} / \mathrm{dL}$ group to $55.4 \%$ of those in the $>130 \mathrm{mg} / \mathrm{dL}$ group. Use of antidiabetic medication was observed in $27.3 \%$ of the study sample. Very few patients used antiarrhythmics $(0.4 \%-0.5 \%$; Table 1$)$.

The ASCVD + probable HeFH subgroup had a mean (SD) age of 65.3 (11.6) years, and $66.1 \%$ of them were female (Table 2). The comorbidity profile of this subgroup suggested few serious comorbidities (mean [SD] CCI: 0.01 [0.09]; 99\% with $\mathrm{CCI}=0$ ); however, a high proportion of these patients had hypertension (62.7\%). Most of these patients used antihypertensives $(56.2 \%)$ but use of antidiabetics (16.3\%) was lower. Few patients were on antiarrhythmics $(0.3 \%)$. The mean (SD) index LDL-C for this subgroup was 215.97 (30.92; Table 2).

In terms of prescriptions for LLT, of the final study sample ( $\mathrm{n}=186,670$; LDL-C $>70 \mathrm{mg} / \mathrm{dL}$ at baseline), only $40.5 \%(\mathrm{n}=75,523)$ had prior LLT treatment at baseline (Figure 1); the majority (92.1\%) received statins only; $2.4 \%$ were receiving ezetimibe only; and $5.5 \%$ were receiving statin + ezetimibe. Prior LLT treatment was also not common in patients with $\mathrm{HeFH}$; of the 4,137 HeFH sample, only $1,073(25.1 \%)$ were previously treated (Table 5).

\section{LDL-C goal achievement in ASCVD patients} Untreated patients initiating post-index therapy

Of the overall study sample that were untreated at baseline $(n=111,147)$, only $34.8 \%(n=38,684)$ initiated treatment in the post-index period, with the vast majority $(94.9 \%$, $\mathrm{n}=36,716)$ initiating statins alone, $3.0 \%(\mathrm{n}=1,177)$ initiating ezetimibe alone, and only $2.0 \%(\mathrm{n}=791)$ initiating ezetimibe in combination with a statin (Table 3, Figure 1).

Among all patients initiating treatment post-index, $60.9 \%$ $(\mathrm{n}=23,550)$ had an LDL-C assessment post-treatment; among these, less than one fifth $(19.1 \%, n=4,501)$ achieved an LDL-C $\leq 70 \mathrm{mg} / \mathrm{dL}$ after initiating treatment (Table 3). The rate of goal achievement (LDL-C $\leq 70 \mathrm{mg} / \mathrm{dL}$ ) in patients with a baseline LDL-C $71-100 \mathrm{mg} / \mathrm{dL}$ ranged from $25.0 \%$ in patients initiating ezetimibe to $31.8 \%$ in patients initiating statins and ezetimibe together. Rate of goal achievement decreased with higher baseline LDL-C. For example, in patients initiating statins only, the rates ranged from $16.5 \%$ in patients with a baseline LDL-C $101-130 \mathrm{mg} / \mathrm{dL}$ to only $10.0 \%$ in those with a baseline LDL-C over $>130 \mathrm{mg} / \mathrm{dL}$. A similar 


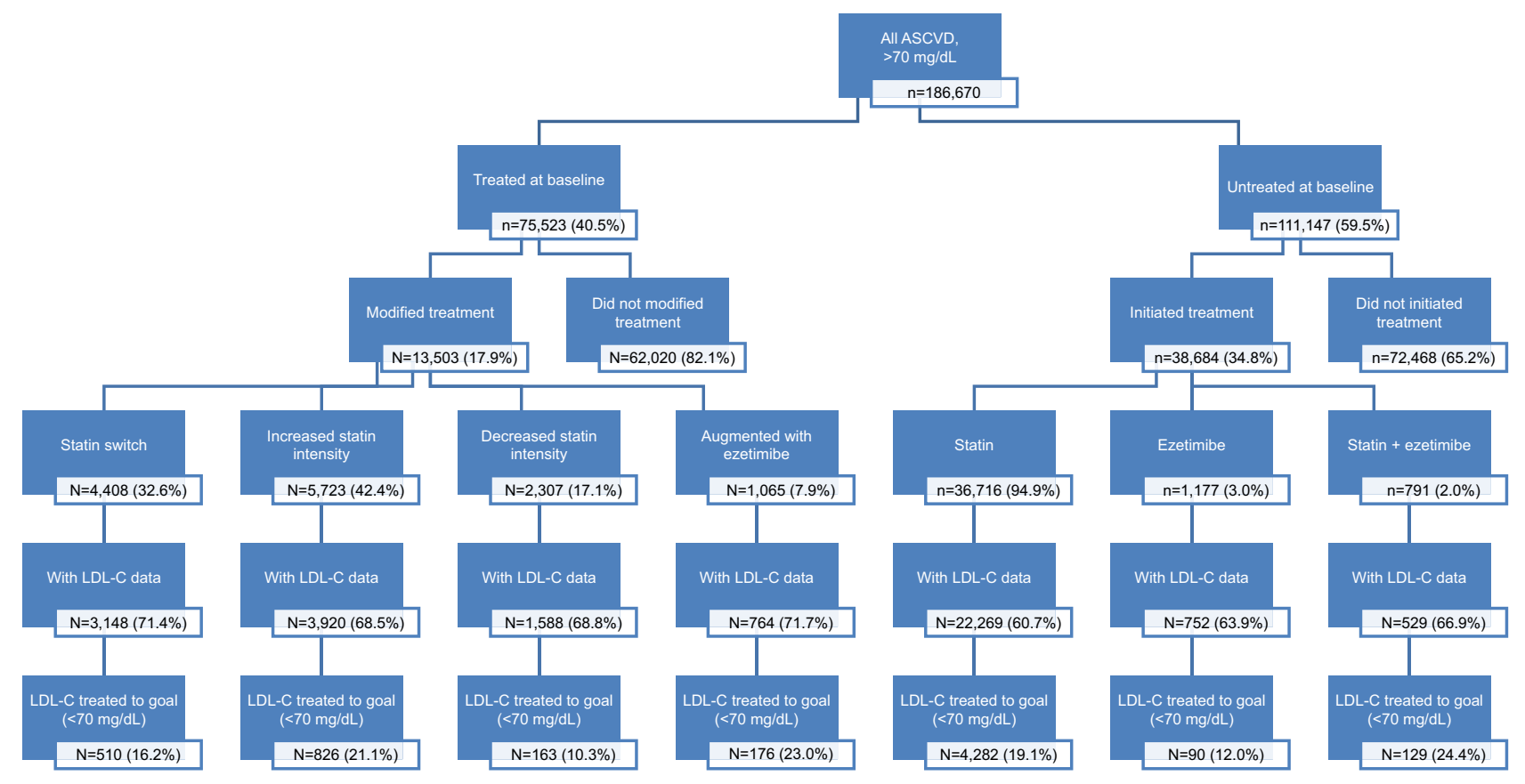

Figure I Summary of post-index treatment and goal achievement, by baseline treated vs not treated.

Abbreviations: ASCVD, atherosclerotic cardiovascular disease; LDL-C, low-density lipoprotein cholesterol.

Table 3 Goal achievement in untreated ASCVD patients initiating treatment

\begin{tabular}{|c|c|c|c|c|}
\hline \multirow{2}{*}{$\begin{array}{l}\text { Cohort } \\
\text { Index LDL-C value }\end{array}$} & \multicolumn{4}{|c|}{ ASCVD patients, untreated at index, $N=\mid$ I I, 147} \\
\hline & $\begin{array}{l}\text { All patients } \\
\mathrm{N}=1 \mathrm{I}, 147(100.0 \%)\end{array}$ & $\begin{array}{l}7 \mid-100 \mathrm{mg} / \mathrm{dL} \\
\mathrm{n}=47,882(43.1 \%)\end{array}$ & $\begin{array}{l}10 \mid-130 \mathrm{mg} / \mathrm{dL} \\
\mathrm{n}=34,983(31.5 \%)\end{array}$ & $\begin{array}{l}>130 \mathrm{mg} / \mathrm{dL} \\
\mathrm{n}=\mathbf{2 8 , 2 8 2}(25.5 \%)\end{array}$ \\
\hline Pts initiating LLT therapy, $\mathrm{n}(\%)$ & $38,684(34.8)$ & $17,078(35.7)$ & $10,56 \mid(30.2)$ & II,045 (39.1) \\
\hline Pts with post-therapy LDL-C, n (\%) & $23,550(60.9)$ & $10,389(60.8)$ & $6,474(61.3)$ & $6,687(60.5)$ \\
\hline Pts with $\geq 50 \%$ LDL-C reduction, $\%(\mathrm{Cl})$ & $7.6(7.3-8.0)$ & $1.8(1.6-2.1)$ & $6.8(6.2-7.4)$ & $17.4(16.5-18.3)$ \\
\hline Pts with LDL-C $\leq 70$ mg/dL, \% (Cl) & $19.1(18.6-19.6)$ & $26.9(26.0-27.7)$ & $16.4(15.5-17.3)$ & $9.7(8.9-10.4)$ \\
\hline LDL-C, mean (SD) & $96.29(32.30)$ & $83.61(22.94)$ & $96.74(28.02)$ & I I 5.54 (38.42) \\
\hline Pts initiating statin only, $\mathrm{n}(\%)$ & $36,716(94.9)$ & $16,28 \mid(95.3)$ & $10,05 \mid(95.2)$ & 10,384 (94.I) \\
\hline Pts with post-therapy LDL-C, n (\%) & $22,269(60.7)$ & $9,878(60.7)$ & $6,132(61.0)$ & $6,259(60.3)$ \\
\hline Pts with $\geq 50 \%$ LDL-C reduction, $\%(\mathrm{Cl})$ & $7.8(7.4-8.1)$ & $1.9(1.6-2.1)$ & $6.9(6.2-7.5)$ & $17.9(17.0-18.9)$ \\
\hline Pts with LDL-C $\leq 70$ mg/dL, \% (Cl) & $19.1(18.7-19.7)$ & $26.8(25.9-27.7)$ & $16.5(15.6-17.4)$ & $10.0(9.3-10.7)$ \\
\hline LDL-C, mean (SD) & $95.78(31.93)$ & $83.62(22.87)$ & $96.39(27.94)$ & II $4.37(38.18)$ \\
\hline Pts initiating ezetimibe only, $\mathrm{n}(\%)$ & I, $177(3.0)$ & $332(1.9)$ & $339(3.2)$ & $506(4.6)$ \\
\hline Pts with post-therapy LDL-C, n (\%) & $752(63.9)$ & $200(60.2)$ & $224(66.1)$ & $328(64.8)$ \\
\hline Pts with $\geq 50 \%$ LDL-C reduction, $\%(\mathrm{Cl})$ & $4.8(3.3-6.3)$ & $2.0(0.0-4.0)$ & $6.3(3.1-9.4)$ & $5.5(3.0-8.0)$ \\
\hline Pts with LDL-C $\leq 70$ mg/dL, \% (Cl) & $12.0(9.6-14.3)$ & $25.0(|8.9-3| . \mid)$ & $13.8(9.3-18.4)$ & $2.7(1.0-4.5)$ \\
\hline LDL-C, mean (SD) & $114.96(38.64)$ & $87.31(27.26)$ & $104.15(28.38)$ & I39.20 (35.93) \\
\hline Pts initiating statin + ezetimibe, $\mathrm{n}(\%)$ & $791(2.0)$ & $465(2.72)$ & I7I (1.6) & $155(1.4)$ \\
\hline Pts with post-therapy LDL-C, n (\%) & $529(66.9)$ & $311(66.88)$ & $118(69.01)$ & $100(64.52)$ \\
\hline Pts with $\geq 50 \%$ LDL-C reduction, $\%(\mathrm{Cl})$ & $4.9(4.7-9.0)$ & $1.0(-0.1-2.1)$ & $5.1(1.1-9.1)$ & $27.0(\mid 8.1-35.9)$ \\
\hline Pts with LDL-C $\leq 70$ mg/dL, \% (Cl) & $24.4(20.7-28.1)$ & $31.8(26.6-37.0)$ & $17.0(10.1-23.8)$ & $10.0(4.0-16.0)$ \\
\hline LDL-C, mean (SD) & 91.19 (29.59) & $81.15(21.86)$ & I00.7I (29.7I) & III.I8 (36.07) \\
\hline
\end{tabular}

Notes: Only patients with a recorded valid LDL-C measurement from I to 12 months after new treatment initiation are shown. The immediate test value post-treatment initiation was used (test values were limited to those observed between I and I 2 months post-treatment initiation).

Abbreviations: ASCVD, atherosclerotic cardiovascular disease; LDL-C, low-density lipoprotein cholesterol; LLT, lipid-lowering therapy; Pts, patients. 
trend was observed in patients initiating ezetimibe alone and in those initiating statin + ezetimibe combination (Table 3 ).

Overall, only $7.8 \%$ of patients achieved a $\geq 50 \%$ reduction in LDL-C post-treatment initiation, and the rate appeared to vary by baseline LDL-C level and the type of treatment initiated (from $1.0 \%$ in the subgroup of patients with index LDL-C $71-100 \mathrm{mg} / \mathrm{dL}$ and initiating statin + ezetimibe to $27.0 \%$ in the subgroup of patients with index LDL-C $>130 \mathrm{mg} / \mathrm{dL}$ and initiating statin + ezetimibe) (Table 3 ).

\section{Treated patients modifying therapy post-index}

Among all treated ASCVD patients with LDL-C $>70 \mathrm{mg} / \mathrm{dL}$ at index $(n=75,523), 17.9 \%(n=13,503)$ received post-index treatment modification, with the most common modification being change in statin dose intensity $(59.5 \%)$, followed by statin switching (32.6\%), and augmentation of statin therapy (7.9\%) post-index (Table 4, Figure 1). There was variation in the rates of treatment modifications across the three baseline LDL-C subgroups as described in Table 4, with modifications more common in patients with LDL-C $>130 \mathrm{mg} / \mathrm{dL}(34.6 \%$, $\mathrm{n}=3,340)$. Among patients modifying therapy post-index, LDL-C goal achievement can only be evaluated in the $69.8 \%(n=9,240)$ with an LDL-C assessment post-treatment modification.

LDL-C outcomes (rates of $>50 \%$ reduction in LDL-C and LDL-C $<70 \mathrm{mg} / \mathrm{dL}$ ) were also found to be suboptimal in

Table 4 Goal achievement in treated ASCVD patients with post-index therapy modification

\begin{tabular}{|c|c|c|c|c|}
\hline \multirow{2}{*}{$\begin{array}{l}\text { Cohort } \\
\text { Index LDL-C value }\end{array}$} & \multicolumn{4}{|c|}{ ASCVD patients, treated at index $N=75,523$} \\
\hline & $\begin{array}{l}\text { All patients } \\
\mathrm{N}=\mathbf{7 5}, \mathbf{5 2 3}(100.0 \%)\end{array}$ & $\begin{array}{l}7 \mathrm{I}-100 \mathrm{mg} / \mathrm{dL} \\
\mathrm{N}=46,44 \mid(6 \mid .5 \%)\end{array}$ & $\begin{array}{l}10|-| 30 \mathrm{mg} / \mathrm{dL} \\
\mathrm{N}=|9,44|(25.7 \%)\end{array}$ & $\begin{array}{l}>130 \mathrm{mg} / \mathrm{dL} \\
\mathrm{N}=9,64 \mid(12.8 \%)\end{array}$ \\
\hline Pts modifying therapy, n (\%) & 13,503 (I7.9) & $5,723(12.3)$ & $4,440(22.8)$ & $3,340(34.6)$ \\
\hline Pts with post-therapy LDL-C, n (\%) & $9,420(69.8)$ & $4,044(70.7)$ & $3,120(70.3)$ & $2,256(67.5)$ \\
\hline Pts with $\geq 50 \%$ LDL-C reduction, $\%(\mathrm{Cl})$ & $5.6(5.1-6.0)$ & I.8 (I.4-2.2) & $4.6(3.9-5.4)$ & $13.6(12.2-15.0)$ \\
\hline Pts with LDL-C $\leq 70$ mg/dL, \% (Cl) & $17.8(17.0-18.6)$ & $26.7(25.4-28.1)$ & $14.0(\mid 2.8-15.3)$ & $6.9(5.9-8.0)$ \\
\hline LDL-C, mean (SD) & $99.03(34.59)$ & $86.50(26.39)$ & $99.28(29.85)$ & $121.17(41.76)$ \\
\hline Pts switching statin, n (\%) & $4,408(32.6)$ & $2,012(35.2)$ & $1,366(30.8)$ & I,030 (30.8) \\
\hline Pts with post-therapy LDL-C, n (\%) & $3,148(7 \mid .4)$ & I,462 (72.7) & 996 (72.9) & $690(67.0)$ \\
\hline Pts with $\geq 50 \%$ LDL-C reduction, $\%(\mathrm{Cl})$ & $5.4(4.6-6.2)$ & $2.0(1.3-2.7)$ & $4.1(2.9-5.4)$ & $14.6(12.0-17.3)$ \\
\hline Pts with LDL-C $\leq 70$ mg/dL, \% (Cl) & $16.2(14.9-17.5)$ & $23.3(21.2-25.5)$ & $12.2(10.1-14.2)$ & $7.0(5.1-8.9)$ \\
\hline LDL-C, mean (SD) & 99.47 (33.99) & $87.32(25.09)$ & $101.53(29.97)$ & $122.24(42.47)$ \\
\hline Pts increasing statin intensity, $\mathrm{n}(\%)$ & $5,723(42.4)$ & $2,156(37.7)$ & $2,116(47.7)$ & $\mathrm{I}, 45 \mathrm{I}(43.4)$ \\
\hline Pts with post-therapy LDL-C, $n$ (\%) & $3,920(68.5)$ & $\mathrm{I}, 485(68.9)$ & $1,472(69.6)$ & $963(66.4)$ \\
\hline Pts with $\geq 50 \%$ LDL-C reduction, $\%(\mathrm{Cl})$ & $6.6(5.8-7.3)$ & $2.1(1.4-2.8)$ & $5.2(4.1-6.4)$ & $15.5(13.2-17.8)$ \\
\hline Pts with LDL-C $\leq 70$ mg/dL, \% (Cl) & $21.1(19.8-22.3)$ & $34.5(32.1-36.9)$ & $16.0(14.1-17.8)$ & $8.2(6.5-9.9)$ \\
\hline LDL-C, mean (SD) & $94.79(33.10)$ & $80.56(23.52)$ & $94.52(27.00)$ & $117.13(41.02)$ \\
\hline Pts decreasing statin intensity, $\mathrm{n}(\%)$ & $2,307(17.1)$ & $\mathrm{I}, \mathrm{I} 46(20.0)$ & $604(13.6)$ & $557(16.7)$ \\
\hline Pts with post-therapy LDL-C, n (\%) & I,588 (68.8) & $794(69.3)$ & $402(66.6)$ & $392(70.4)$ \\
\hline Pts with $\geq 50 \%$ LDL-C reduction, $\%(\mathrm{Cl})$ & $3.2(2.3-4.0)$ & $0.5(0.0-1.0)$ & $4.2(2.3-6.2)$ & $7.4(4.8-10.0)$ \\
\hline Pts with LDL-C $\leq 70$ mg/dL, \% (Cl) & $10.3(8.8-11.8)$ & $13.6(\mid 1.2-16.0)$ & $10.5(7.4-13.5)$ & $3.3(1.5-5.1)$ \\
\hline LDL-C, mean (SD) & $108.70(36.50)$ & $97.70(29.28)$ & III.25 (34.53) & $128.37(42.49)$ \\
\hline Pts augmenting with ezetimibe, $\mathrm{n}(\%)$ & I,065 (7.9) & $409(7.2)$ & $354(8.0)$ & $302(9.0)$ \\
\hline Pts with post-therapy LDL-C, n (\%) & $764(71.7)$ & $303(74.1)$ & $250(70.6)$ & $211(69.9)$ \\
\hline Pts with $\geq 50 \%$ LDL-C reduction, $\%(\mathrm{Cl})$ & $6.0(4.3-7.7)$ & $3.0(1.0-4.9)$ & $3.6(1.3-5.9)$ & $13.3(8.7-17.9)$ \\
\hline Pts with LDL-C $\leq 70$ mg/dL, \% (Cl) & $23.0(20.0-26.0)$ & $39.6(34.1-45.1)$ & $16.0(11.4-20.6)$ & $7.6(4.0-11.2)$ \\
\hline LDL-C, mean (SD) & $98.92(36.35)$ & $82.23(28.04)$ & $99.06(31.01)$ & 122.73 (39.46) \\
\hline
\end{tabular}

Notes: Only patients with a recorded valid LDL-C measurement from I to 12 months after new treatment initiation are shown. The immediate test value post-treatment initiation was used (test values were limited to those observed between I and 12 months post-treatment initiation). Baseline statin intensity: among the 75,523 ASCVD patients treated at baseline, $12.18 \%$ were on low statin intensity, $58.4 \%$ were on moderate statin intensity, $20.6 \%$ were on high statin intensity, and $8.8 \%$ were treated with other lipid-lowering agents.

Abbreviations: ASCVD, atherosclerotic cardiovascular disease; LDL-C, low-density lipoprotein cholesterol; Pts, patients. 
Table 5 Goal achievement in probable HeFH patients who initiated or modified treatment post-index

\begin{tabular}{|c|c|c|c|}
\hline $\begin{array}{l}\text { Probable HeFH patients (index } \\
\text { LDL-C value } \geq 190 \mathrm{mg} / \mathrm{dL} \text { ) } \\
\text { (untreated at index) }\end{array}$ & $n=3,064(2.8 \%)$ & $\begin{array}{l}\text { Probable HeFH patients (index } \\
\text { LDL-C value } \geq 190 \mathrm{mg} / \mathrm{dL} \text { ) } \\
\text { (treated at index) }\end{array}$ & $\mathrm{n}=\mathrm{I}, 073(1.4 \%)$ \\
\hline Pts initiating LLT therapy, $\mathrm{n}(\%)$ & $\mathrm{I}, 540(50.3)$ & Pts modifying therapy, $\mathrm{n}(\%)$ & $445(4 I .5)$ \\
\hline Pts with post-therapy LDL-C, n (\%) & $896(58.2)$ & Pts with post-therapy LDL-C, n (\%) & $28 I(63.2)$ \\
\hline Pts with $\geq 50 \%$ LDL-C reduction, $\%(\mathrm{Cl})$ & $31 . I(28.1-34.2)$ & Pts with $\geq 50 \%$ LDL-C reduction, $\%$ & $22.1(17.2-26.9)$ \\
\hline Pts with LDL-C $\leq 70$ mg/dL, $\%(C l)$ & $4.4(3.0-5.7)$ & Pts with LDL-C $\leq 70 \mathrm{mg} / \mathrm{dL}, \%$ & I.I $(-0.1-2.3)$ \\
\hline LDL-C, mean (SD) & $|36.2|(47.75)$ & LDL-C, mean (SD) & $151.87(54.37)$ \\
\hline Pts initiating statin only, $\mathrm{n}(\%)$ & $1,410(91.6)$ & Pts switching statin, $\mathrm{n}(\%)$ & $153(34.4)$ \\
\hline Pts with post-therapy LDL-C, n (\%) & $815(57.8)$ & Pts with post-therapy LDL-C, n (\%) & 81 (52.9) \\
\hline Pts with $\geq 50 \%$ LDL-C reduction, $\%(\mathrm{Cl})$ & $32.0(28.8-35.2)$ & Pts with $\geq 50 \%$ LDL-C reduction, $\%$ & $22.2(|3.0-3| .5)$ \\
\hline Pts with LDL-C $\leq 70$ mg/dL, \% (Cl) & $4.8(3.3-6.3)$ & Pts with LDL-C $\leq 70 \mathrm{mg} / \mathrm{dL}, \%$ & 0.0 \\
\hline LDL-C, mean (SD) & $135.12(48.04)$ & LDL-C, Mean (SD) & $147.40(49.25)$ \\
\hline Pts initiating ezetimibe only, $\mathrm{n}(\%)$ & $90(5.8)$ & Pts increasing statin intensity, $\mathrm{n}(\%)$ & $170(38.2)$ \\
\hline Pts with post-therapy LDL-C, n (\%) & $58(64.4)$ & Pts with post-therapy LDL-C, n (\%) & $109(64.1)$ \\
\hline Pts with $\geq 50 \%$ LDL-C reduction, $\%(\mathrm{Cl})$ & $10.3(2.3-18.4)$ & Pts with $\geq 50 \%$ LDL-C reduction, $\%$ & $26.6(18.2-35.0)$ \\
\hline Pts with LDL-C $\leq 70$ mg/dL, \% (Cl) & 0.0 & Pts with LDL-C $\leq 70 \mathrm{mg} / \mathrm{dL}, \%$ & $1.8(-0.7-4.4)$ \\
\hline LDL-C, mean (SD) & $110.93(32.07)$ & LDL-C, mean (SD) & $152.55(57.84)$ \\
\hline Pts initiating statin + ezetimibe, $n(\%)$ & $40(2.6)$ & Pts decreasing statin intensity, n (\%) & $81(18.2)$ \\
\hline Pts with post-therapy LDL-C, n (\%) & $23(57.50)$ & Pts with post-therapy LDL-C, n (\%) & $61(75.3)$ \\
\hline Pts with $\geq 50 \%$ LDL-C reduction, $\%(\mathrm{Cl})$ & $52.2(30.1-74.3)$ & Pts with $\geq 50 \%$ LDL-C reduction, $\%$ & I3.I (4.4-2I.8) \\
\hline Pts with LDL-C $\leq 70$ mg/dL, \% (Cl) & 0.0 & Pts with LDL-C $\leq 70 \mathrm{mg} / \mathrm{dL}, \%$ & 0.0 \\
\hline LDL-C, mean (SD) & $161.45(38.89)$ & LDL-C, mean (SD) & I $64.49(57.70)$ \\
\hline
\end{tabular}

Notes: Only patients with a recorded valid LDL-C measurement from I to 12 months after new treatment initiation are shown. The immediate test value post-treatment initiation was used (test values were limited to those observed between I and 12 months post-treatment initiation). Baseline statin intensity: among the I,073 probable HeFH patients treated at baseline, $10.7 \%$ were on low statin intensity, $48.6 \%$ were on moderate statin intensity, $24.6 \%$ were on high statin intensity, and $16.0 \%$ were treated with other LLTs. Abbreviations: HeFH, heterozygous familial hypercholesterolemia; LDL-C, low-density lipoprotein cholesterol; LLT, lipid-lowering therapy; Pts, patients.

treated patients who modified therapy post-index as described in Table 4. The most effective treatment modification overall was adding ezetimibe. Among patients with baseline LDL-C $71-100 \mathrm{mg} / \mathrm{dL}, 39.6 \%$ achieved the LDL-C $\leq 70 \mathrm{mg} / \mathrm{dL}$ threshold after adding ezetimibe, compared to only $16.0 \%$ and $7.5 \%$ in patients with baseline LDL-C $101-130 \mathrm{mg} / \mathrm{dL}$ and $>130 \mathrm{mg} / \mathrm{dL}$, respectively. Rates of goal achievement were similar for patients who increased their statin intensity, but lower for patients increasing the dose of the index statin. The proportion of patients achieving a $\geq 50 \%$ reduction in baseline LDL-C were highest in those with baseline LDL-C $>130 \mathrm{mg} / \mathrm{dL}$, and ranged from $7.4 \%$ (decreased statin intensity) to $15.5 \%$ (increased statin intensity) (Table 4).

\section{LDL-C goal achievement in probable HeFH patients}

Untreated patients initiating post-index therapy

In the probable HeFH subgroup of patients untreated at baseline $(\mathrm{n}=3,064)$, half of the patients $(50.3 \%, \mathrm{n}=1,540)$ initiated treatment post-index. The mean (SD) post-treatment LDL-C was $136.2(47.8) \mathrm{mg} / \mathrm{dL}$ and post-treatment LDL-C was lowest for those initiating ezetimibe (110.9 [32.1] mg/dL). Overall, only $4.8 \%$ of these patients initiating statin achieved the LDL-C $\leq 70 \mathrm{mg} / \mathrm{dL}$ threshold. None of the ASCVD + probable $\mathrm{HeFH}$ patients initiating ezetimibe only, or statin + ezetimibe achieved goal LDL-C threshold. Approximately $31.1 \%$ of these patients had a $\geq 50 \%$ reduction in LDL-C after initiating therapy; $50 \%$ reduction occurred most frequently in patients initiating statin + ezetimibe (52.2\%) (Table 5).

\section{Treated patients modifying therapy post-index}

Only 1,073 probable $\mathrm{HeFH}$ patients treated at baseline and a large majority received statin alone. Of those treated, $41.5 \%$ $(n=445)$ modified treatment. Based on the 281 patients with available post-treatment LDL-C assessment, overall 62 patients (22.1\%) achieved a $\geq 50 \%$ reduction in LDL-C, but only $3(1.1 \%)$ patients reached a LDL-C of $\leq 70 \mathrm{mg} / \mathrm{dL}$. The highest $\geq 50 \%$ LDL-C reduction was observed in the patients increasing statin intensity at $26.61 \%$ (Table 5).

\section{Discussion}

In the current study, a majority (59.5\%) of the patients with a diagnosis of ASCVD and an LDL-C $>70 \mathrm{mg} / \mathrm{dL}$ were 
not previously or currently receiving statin or ezetimibe therapy at the time the baseline abnormal LDL-C level was observed. As we indexed patients to their first LDL-C test during the index window, it is possible that for some patients the baseline LDL-C assessment was their first abnormal one. Nevertheless, this finding of many untreated patients corroborates the observation by other researchers who postulated that despite evidence showing statins and ezetimibe to reduce the risk of ASCVD-related events, many patients do not receive treatment per established guidelines. ${ }^{10,23,24}$ It has been suggested that provider confusion regarding the most appropriate guidelines for a patient and attempting to implement recommendations that are evolving may contribute to this treatment gap. ${ }^{10}$

The rate of treatment initiation among previously untreated patients was generally under $40 \%$. In patients with an LDL-C >190 mg/dL (probable HeFH patients), the treatment initiation rate was higher, but still only at approximately $50 \%$. Rates of treatment modification after elevated index LDL-C values were also low overall, ranging from a low of $12.3 \%$ in patients with a baseline LDL-C $71-100 \mathrm{mg} / \mathrm{dL}$ to $34.6 \%$ in patients with a baseline LDL-C $>130 \mathrm{mg} / \mathrm{dL}$. An earlier study entailing a survey of health care providers also indicated that it was not uncommon for LLT to remain unchanged, even when the patient is not at goal. That survey found that while the need for lower LDL-C and CV risk was considered important in these patients by the health care providers, physicians' treatment choices were still substantially less aggressive than guideline recommendations. ${ }^{25}$

Although there was an overall decrease in the mean LDL-C value among previously untreated ASCVD patients initiating therapy post-index, the majority $(80.9 \%)$ did not achieve the LDL-C threshold of $\leq 70 \mathrm{mg} / \mathrm{dL}$. This should not be interpreted as a reason not to initiate treatment, but rather to titrate patients to higher intensity statins, and add ezetimibe per guidelines, to increase the chance of achieving an LDL-C $\leq 70 \mathrm{mg} / \mathrm{dL}$. If still not successful in achieving treatment goals, initiating alternative agents such as PCSK9 inhibitors may be warranted.

LDL-C treatment outcomes in treated patients who modified treatment were also not optimal. Current analysis observed a general trend that patients with higher baseline LDL-C values had greater post-modification LDL-C reductions. In addition, post-modification benefits were more common in patients who increased statin dose intensity than in those who switched or augmented therapy. However, even in these subgroups of patients who showed greater post-treatment LDL-C reduction, the majority of them were still not at goal ( $\leq 70 \mathrm{mg} / \mathrm{dL}$, or $50 \%$ reduction) based on the first LDL-C test post-treatment change. These findings are consistent with other published reports. ${ }^{26,27}$ The EUROASPIRE surveys found that in the past years, although the use of LLTs, including high-intensity statins, increased, the LDL-C treatment outcome is still suboptimal. ${ }^{26,27}$

This study underscores the challenges of managing LDL-C levels in patients with ASCVD and probable $\mathrm{HeFH}$ and provides further evidence of the need for more aggressive treatment, including high-intensity statin and alternative therapies, for this population. In addition, barriers such as utilization management that require patients and providers to navigate payer-imposed utilization management tasks related to step-therapy or prior authorization may inadvertently delay patients in receiving therapies that may support achieving therapeutic goals.

\section{Limitations}

These findings should be interpreted in the context of specific limitations of the study. The use of EMR data may have introduced some information bias as only data from physicians contributing to the EMR network were available. In addition, EMR data are not created for research purposes, and their quality is subject to a tradeoff between data entry and patient care. Patient visits conducted by other health care providers not included in the EMR data network were not captured, which may have led to the underreporting of LDL-C measurements and/or treatment with LLTs. Treatment exposure to statin and/or ezetimibe was based on observation of a valid prescription recorded in the EMR database, which does not guarantee that the patient filled the prescription or used the medication. Incomplete LDL-C data capture in the EMR system may also limit the generalizability of LDL-C treatment outcomes observed in this study. In addition, age at initial ASCVD diagnosis cannot be confirmed in this database, thus limiting the interpretation of the LLT treatment patterns and outcomes observed in a prevalent population. This study defined probable $\mathrm{HeFH}$ as with baseline LDL-C level $\geq 190 \mathrm{mg} / \mathrm{dL}$, not by confirmed clinical diagnosis or genetic tests; this may lead to false positive/negative $\mathrm{HeFH}$ in identification of these patients in our study sample. Finally, this was a descriptive retrospective analysis that evaluates the associations between exposures and outcomes, but no causal relationships can be established from this observational study.

\section{Conclusion}

This study substantiates previous reports of under-prescribing of LLTs to ASCVD patients with elevated LDL-C levels. 
Moreover, for most of the patients with ASCVD and probable $\mathrm{HeFH}$, standard treatment regimens or modifications of recommended treatments failed to produce $>50 \%$ reduction in LDL-C and/or achieve LDL-C values of $<70 \mathrm{mg} / \mathrm{dL}$ for most patients, regardless of their baseline LDL-C levels. More frequent and aggressive lipid management, including increasing statin intensity and alternative therapies, may be needed in these patients to reduce their CV risk. Given this and other evidence such as findings of high discontinuation rates for statin therapy, particularly for high-intensity statin doses, new health care system-based interventions and therapeutic paradigms are needed to address the unmet need in ASCVD and probable HeFH patients with elevated LDL-C levels.

\section{Acknowledgments}

Jin Wu (Statistical Programmer, IQVIA) provided analytic support. Xin Wang (consultant, IQVIA) provided editorial support for this manuscript. This study was sponsored by Amgen Inc.

\section{Disclosure}

C. C. Chen, D.M. Hines, and R.L. Wade are employees of IQVIA. IQVIA was hired by Amgen Inc. to conduct this study. P.B. Rane, J. Patel, J., and D. J Harrison are employees and stockholders of Amgen Inc.

\section{References}

1. Centers for Disease Control and Prevention, National Center for Health Statistics [webpage on the Internet]. Underlying Cause of Death 1999-2014: Multiple Cause of Death Files (1999-2014), 2015. Available from: https://wonder.cdc.gov/ucd-icd10.html. Accessed November 2, 2016.

2. Kullo IJ, Trejo-Gutierrez JF, Lopez-Jimenez F, et al. A perspective on the New American College of Cardiology/American Heart Association guidelines for cardiovascular risk assessment. Mayo Clin Proc. 2014; 89(9):1244-1256.

3. Catapano AL, Graham I, De Backer G, et al. 2016 ESC/EAS Guidelines for the Management of Dyslipidaemias. Eur Heart J. 2016;37(39): 2999-3058.

4. Najam O, Ray KK. Familial hypercholesterolemia: a review of the natural history, diagnosis, and management. Cardiol Ther. 2015;4(1): $25-38$.

5. Reiner Ž. Management of patients with familial hypercholesterolaemia. Nat Rev Cardiol. 2015;12(10):565.

6. Stone NJ, Robinson JG, Lichtenstein AH, et al. 2013 ACC/AHA guideline on the treatment of blood cholesterol to reduce atherosclerotic cardiovascular risk in adults: a report of the American College of Cardiology/American Heart Association Task Force on Practice Guidelines. $J$ Am Coll Cardiol. 2014;63(25 Pt B):2889-2934.

7. Foody JM, Sajjan SG, Hu XH, et al. Loss of early gains in low-density lipoprotein cholesterol goal attainment among high-risk patients. $J$ Clin Lipidol. 2010;4(2):126-132.

8. Nichols GA, Nag S, Chan W. Intensity of lipid-lowering therapy and low-density lipoprotein cholesterol goal attainment among the elderly before and after the 2004 National Cholesterol Education Program Adult Treatment Panel III update. Am Heart J. 2007;154(3):554-560.
9. Foody JM, Toth PP, Tomassini JE, et al. Changes in LDL-C levels and goal attainment associated with addition of ezetimibe to simvastatin, atorvastatin, or rosuvastatin compared with titrating statin monotherapy. Vasc Health Risk Manag. 2013;9:719-727.

10. Morris PB, Ballantyne CM, Birtcher KK, Dunn SP, Urbina EM. Review of clinical practice guidelines for the management of LDL-related risk. J Am Coll Cardiol. 2014;64(2):196-206.

11. Lin I, Sung J, Sanchez RJ, et al. Patterns of Statin Use in a Real-World Population of Patients at High Cardiovascular Risk. J Manag Care Spec Pharm. 2016;22(6):685-698.

12. Expert Panel on Detection, Evaluation, and Treatment of High Blood Cholesterol in Adults. Executive Summary of The Third Report of The National Cholesterol Education Program (NCEP) Expert Panel on Detection, Evaluation, And Treatment of High Blood Cholesterol In Adults (Adult Treatment Panel III). JAMA. 2001;285(19):2486-2497.

13. Sabatine MS, Giugliano RP, Wiviott SD, et al. Efficacy and safety of evolocumab in reducing lipids and cardiovascular events. $N$ Engl $J$ Med. 2015;372(16):1500-1509.

14. Marrett E, Zhao C, Zhang NJ, et al. Limitations of real-world treatment with atorvastatin monotherapy for lowering LDL-C in high-risk cardiovascular patients in the US. Vasc Health Risk Manag. 2014;10:237-246.

15. Robinson JG, Farnier M, Krempf M, et al. Efficacy and safety of alirocumab in reducing lipids and cardiovascular events. $N$ Engl J Med. 2015;372(16):1489-1499.

16. Phan K, Gomez YH, Elbaz L, Daskalopoulou SS. Statin treatment non-adherence and discontinuation: clinical implications and potential solutions. Curr Pharm Des. 2014;20(40):6314-6324.

17. Caspard H, Chan AK, Walker AM. Compliance with a statin treatment in a usual-care setting: retrospective database analysis over 3 years after treatment initiation in health maintenance organization enrollees with dyslipidemia. Clin Ther. 2005;27(10):1639-1646.

18. Virani SS, Woodard LD, Akeroyd JM, Ramsey DJ, Ballantyne CM, Petersen LA. Is high-intensity statin therapy associated with lower statin adherence compared with low- to moderate-intensity statin therapy? Implications of the 2013 American College of Cardiology/American Heart Association Cholesterol Management Guidelines. Clin Cardiol. 2014;37(11):653-659.

19. Writing Committee, Lloyd-Jones DM, Morris PB, et al. 2016 ACC Expert Consensus Decision Pathway on the Role of Non-Statin Therapies for LDL-Cholesterol Lowering in the Management of Atherosclerotic Cardiovascular Disease Risk: A Report of the American College of Cardiology Task Force on Clinical Expert Consensus Documents. $J$ Am Coll Cardiol. 2016;68(1):92-125.

20. Duke JD, Ryan PB, Suchard MA, et al. Risk of angioedema associated with levetiracetam compared with phenytoin: Findings of the observational health data sciences and informatics research network. Epilepsia. 2017;58(8):e101-e106.

21. Shafrin J, Tebeka MG, Price K, Patel C, Michaud K. The economic burden of ACPA-positive status among patients with rheumatoid arthritis. J Manag Care Spec Pharm. 2018;24(1):4-11.

22. Mosnaim G, Ariely R, Depietro M. Clinical Outcomes in Patients with Persistent Asthma by Attainment of Healthcare Effectiveness and Data Information Set (HEDIS) Measures. Poster presented at: AAE Annual Conference; July 20-22; 2018; Phoenix, AZ

23. Somma K, Bhatt D, Fonarow G. Guideline adherence after ST-segment elevation versus non-ST segment elevation myocardial infarction. Circ Cardiovasc Interv. 2013;5(5):654-661.

24. Sachdeva A, Cannon CP, Deedwania PC, et al. Lipid levels in patients hospitalized with coronary artery disease: an analysis of 136,905 hospitalizations in Get With The Guidelines. Am Heart J. 2009;157(1):111-117.

25. Krempf M, Simpson RJ, Ramey DR, et al. Patient and physician factors influence decision-making in hypercholesterolemia: a questionnairebased survey. Lipids Health Dis. 2015;14:45.

26. Kotseva K. Lessons from Euroaspire I, II, and III surveys. Heart Metab. 2011;50:32-35.

27. Kotseva K, EUROASPIRE Investigators. The EUROASPIRE surveys: lessons learned in cardiovascular disease prevention. Cardiovasc Diagn Ther. 2017;7(6):633-639. 


\section{Supplementary material}

Table SI Stain dose intensity categories (low, moderate, and high)

\begin{tabular}{|c|c|c|c|c|}
\hline \multirow[t]{2}{*}{ Statin therapy } & \multicolumn{4}{|l|}{ Daily dose } \\
\hline & Low intensity & Moderate intensity & High intensity & Intensity classifications of atypical doses \\
\hline Atorvastatin & $<10 \mathrm{mg} /$ day & 10 to $<40 \mathrm{mg} /$ day & $\geq 40 \mathrm{mg} /$ day & $30 \mathrm{mg} /$ day $=$ moderate \\
\hline Fluvastatin & $<80 \mathrm{mg} /$ day & $80 \mathrm{mg} /$ day & NA & $10 \mathrm{mg} /$ day $=$ low \\
\hline Lovastatin & $<40 \mathrm{mg} /$ day & $\geq 40 \mathrm{mg} /$ day & NA & $\begin{array}{l}10 \mathrm{mg} / \mathrm{day}=\text { low } \\
80 \mathrm{mg} / \mathrm{day}=\text { moderate }\end{array}$ \\
\hline Pitavastatin & $<2 \mathrm{mg} /$ day & $\geq 2 \mathrm{mg} /$ day & NA & \\
\hline Pravastatin & $<40 \mathrm{mg} /$ day & $\geq 40 \mathrm{mg} /$ day & NA & $<10 \mathrm{mg} /$ day $=$ low \\
\hline Rosuvastatin & $<5 \mathrm{mg} /$ day & 5 to $<20 \mathrm{mg} /$ day & $\geq 20 \mathrm{mg} /$ day & $\begin{array}{l}<5 \mathrm{mg} / \text { day }=\text { low } \\
15 \mathrm{mg} / \text { day }=\text { moderate }\end{array}$ \\
\hline Simvastatin & $<20 \mathrm{mg} /$ day & 20 to $<80 \mathrm{mg} /$ day & $\geq 80 \mathrm{mg} /$ day & $\begin{array}{l}<20 \mathrm{mg} / \text { day }=\text { low } \\
>40 \text { to }<80 \mathrm{mg} / \text { day }=\text { moderate } \\
\geq 80 \mathrm{mg} / \text { day }=\text { high }\end{array}$ \\
\hline
\end{tabular}

Abbreviation: NA, not applicable.

\section{Publish your work in this journal}

Therapeutics and Clinical Risk Management is an international, peerreviewed journal of clinical therapeutics and risk management, focusing on concise rapid reporting of clinical studies in all therapeutic areas, outcomes, safety, and programs for the effective, safe, and sustained use of medicines. This journal is indexed on PubMed Central, CAS,
EMBase, Scopus and the Elsevier Bibliographic databases. The manuscript management system is completely online and includes a very quick and fair peer-review system, which is all easy to use. Visit http://www.dovepress.com/testimonials.php to read real quotes from published authors.

Submit your manuscript here: http://www.dovepress.com/therapeutics-and-clinical-risk-management-journal 